\title{
Asymmetric cell division as a route to reduction in cell length and change in cell morphology in trypanosomes.
}

\begin{abstract}
African trypanosomes go through at least five developmental stages during their life cycle. The different cellular forms are classified using morphology, including the order of the nucleus, flagellum and kinetoplast along the anterior-posterior axis of the cell, the predominant cell surface molecules and the location within the host. Here, an asymmetrical cell division cycle that is an integral part of the Trypanosoma brucei life cycle has been characterised in further detail through the use of cell cycle stage specific markers. The cell cycle leading to the asymmetric division includes an exquisitely synchronised mitosis and exchange in relative location of organelles along the anterior-posterior axis of the cell. These events are coupled to a change in cell surface architecture. During the asymmetric division, the behaviour of the new flagellum is consistent with a role in determining the location of the plane of cell division, a function previously characterised in procyclic cells. Thus, the asymmetric cell division cycle provides a mechanism for a change in cell morphology and also an explanation for how a reduction in cell length can occur in a cell shaped by a stable microtubule array.
\end{abstract}

Keyword: Asymmetric cell division; Differentiation; Epimastigote; Trypanosoma brucei. 